Article

\title{
Concept Design and Load Capacity Analysis of a Novel Serial-Parallel Robot for the Automatic Charging of Electric Vehicles
}

\author{
Han Yuan ${ }^{1}$, Qiong $\mathrm{Wu}^{1}$ and Lili Zhou ${ }^{2, *}$ \\ 1 School of Mechanical Engineering and Automation, Harbin Institute of Technology (Shenzhen), \\ Shenzhen 518055, China; yuanhan@hit.edu.cn (H.Y.); 19s153108@stu.hit.edu.cn (Q.W.) \\ 2 School of Automotive and Transportation Engineering, Shenzhen Polytechnic, Shenzhen 518055, China \\ * Correspondence: lilyzhou@szpt.edu.cn
}

Received: 7 May 2020; Accepted: 4 June 2020; Published: 8 June 2020

\begin{abstract}
The automatic charging of electric vehicles is an important but challenging problem. Recently, various charging robots are proposed for electric vehicles. Most previous researches do not pay enough attention to the robots' load capacities. Actually, providing the charging connector with adequate pushing and/or pulling forces is vital to guarantee a reliable electrical connection, which is a key issue for charging robot design. In this paper, we present a novel serial-parallel robot for the automatic charging of electric vehicles. This robot is based on the 3 universal-prismatic-universal (3UPU) parallel mechanism and featured by high-load capacity. We firstly address the kinematic and static models of the proposed robot, then analyze its load capacity. It is shown that the robot's maximum load capacity depends not only on the driving ability of the prismatic joints, but also on the robot's structural parameters and the robot's configuration. Finally, optimizations are made and results show that the robot's load capacity along the desired trajectory has more than doubled. Results of this paper could be useful for the development of automatic electric-vehicle-charging devices.
\end{abstract}

Keywords: serial-parallel robot; high-payload; electric vehicle charging robot; force analysis; load capacity optimization

\section{Introduction}

Electric vehicles (EVs) have attracted plenty of attentions during the past decade as one of the most promising solutions for the energy and environmental crises [1,2]. Until recently, most EV charging devices still have to be operated manually [3,4]. Thus the charging device will be occupied by a single vehicle during its whole parking period, regardless of whether the battery is full or not. This is an urgent problem in many public charging stations, especially for those stations with limited parking places. To solve this problem, automatic EV charging robots are proposed [5-8].

According to the mechanical structure, most existing EV charging robots can be classified into two types. The first type is based on the traditional industrial robot arm, such as the VW e-smart Connect that is based on a KUKA LBR iiwa [5]. These charging robots have a general structure consisting of several rigid joints and links arranged in serial. This structure is simple and mature, but it is not fully suitable for the EV charging due to flowing reasons. Traditional serial robots usually have a low payload to self-weight ratio [9]. They have to be built clumsy to guarantee enough load capacity. In addition, these industrial robots usually have finite degrees of freedom (DOFs), commonly 6 DOFs. Thus the end-effector's dexterity is limited, which is not convenient to plug in and/or pull out the charging connector, especially in confined space with obstacles. Moreover, it is hard to design a serial industrial robot with hollow shaft that has an enough large inner diameter to let the electric wire 
passing through. Therefore, for this type of robots, the charging wire often has to be put outside, which brings difficulties in protecting and arranging the wire. Overall, traditional serial industrial robots are not entirely adapt at the EV charging application due to weak dexterity, limited load capacity and inconvenient cable arrangement, although they are simple and wildly used.

Recently, another type of charging robots is proposed based on cable-driven snake-like manipulators. These manipulators are usually redundantly actuated [10], or even hyper redundant [11,12]. Thus, this type of charging robots could achieve dexterous or even super-dexterous operations in the EV charging application. This characteristic is significant for obstacle avoidance, and thus provides more possibility to plug in the charging connector, especially in confined space. Furthermore, it is not difficult to design a cable-driven snake-like manipulator with hollow structure along its whole arm. In this way, the electric wire can be arranged totally inside the robot arm. However, due to the driving cable elasticity, the stiffness of this type charging robot is low, and thus high positioning accuracy is difficult to be guaranteed $[13,14]$. Furthermore, affected by the friction produced by the sliding movement of driving cables, the actual load capacity of the charging robot could be limited $[15,16]$. Overall, the cable-driven snake-like charging robots have advantages in dexterity and hollow structure, but have inherent weakness in accuracy and load capacity.

To sum up, most existing EV charging robots have limitations in load capacity, and previous literature lacks enough attention to the load capacity analysis. It is unknown whether these charging robots could provide enough pushing and/or pulling forces to the charging connector. In fact, adequate force is vital to guarantee a reliable electrical connection, and this is a key issue for charging robot design. Until now, it is still a challenge to find practical solutions for the design and analysis of EV charging robots.

This paper presents a novel EV charging robot based on hybrid serial-parallel manipulators. In general, the serial-parallel manipulator combines a sequence of parallel mechanisms [17-21]. It has not only the advantages of traditional parallel manipulators such as high load capacity and good stiffness [22-25], but also has the advantages of traditional serial manipulators such as large workspace $[23,26,27]$. This kind of hybrid serial-parallel manipulators has been studied in some precious researches $[17,18,20,28-31]$, but it has not been used in the EV charging robot design as far as we know.

Aiming at the EV charging application, we proposed the concept design of a hybrid serial-parallel robot. This novel EV charging robot consists of three 3 universal-prismatic-universal (3UPU) parallel mechanisms that are arranged in serial. Each 3UPU mechanism has 2 rotational and 1 transnational DOFs, and is driven by 3 prismatic joints. With this design, the charging robot has totally 9 actuating DOFs and achieves 6 DOFs movement of the charging connector that is fixed on the robot end. This robot is redundantly actuated and has a good dexterity to better adjust the connector's posture. In addition, the proposed structure design has a hollow structure that can let the electric wire to pass through.

In addition to the concept design, we also make comprehensive analyses in the load capacity of the proposed EV charging robot. For this purpose, the kinematic and static robot models are deduced, and then validated by the commercial dynamic analysis software Adams (Adams is a widely used multi-body dynamic simulation software by MSC Software Company.). Using the kinematic and static models, we analyze the largest feasible external force applied to the charging connector, along a certain trajectory in the charging procedures. As a result that this largest feasible external force can be used as an index to evaluate the robot's load capacity, it determines whether the robot is strong enough to plug in and/or pull out the charging connector. We find that the largest feasible force is associated with the manipulator configuration due to redundancy, also related to the structural parameters such as the length and diameter of each 3UPU mechanism, and also depends on the actuating ability of the prismatic joints. Based on these results, further optimization is made to maximize the robot's load capacity. It is shown that after optimization, the robot's load capacity along the desired trajectory could be improved by about $130 \%$. A comparison is further made between the 
proposed EV charging robot and its counterparts in literature. Details are shown in Table 1. Results indicate that the proposed new robot has advantages in good dexterity, hollow structure and high load capacity. These characteristics will enable the robots with a good obstacle avoidance ability, also facilitate the electrical wire arrangement, and finally improve the overall performances. In addition to these advantages, the proposed charging robot has a more complex mathematical model due to the hybrid parallel and serial structure. This paper will address the modeling problem of this new type of charging robot to provide a reference for further study.

Table 1. Comparison between the electric vehicles (EV) charging robots in some literature and the proposed robot in this paper.

\begin{tabular}{cccccc}
\hline Type of the EV Charging Robots & DOF & Dexterity & Hollow Structure & Load Capacity & Modeling \\
\hline Industrial robots in [5] & 6 & Poor & No & Normal & Easy \\
Cable-driven snake robots in [10] & 20 & Excellent & Yes & Poor & Normal \\
The proposed serial-parallel robot & 9 & Good & Yes & Good & Difficult \\
\hline
\end{tabular}

The major contributions of this paper are as follows. Firstly, a novel concept is proposed to solve the challenging problem of EV charging robot design. This paper, for the first time so far as we know, introduces the 3UPU based hybrid serial-parallel manipulator to the EV charging robot design. Secondly, the load capacity of the EV charging robot, an important issue which has not attracted enough attentions in literature, is comprehensively analyzed and optimized. Results show 130\% increment in the robot's load capacity after applying the proposed optimization method. The rest of this paper is organized as follows. The concept design and the kinematic model of the EV charging robot are presented in Section 2. Then, the static model is deduced in Section 3. Based on that, simulation validations and load capacity optimizations are made in Section 4. Finally, conclusions are made in Section 5 .

\section{Concept Design and Kinematic Model of the EV Charging Robot}

This section firstly presents the concept design of the EV charging robot. Unlike precious EV charging robots that base on the traditional serial industrial manipulator or the cable-driven serpentine robot, this new design mainly uses the concept of hybrid serial-parallel robots. Then, the kinematic model of the robot is deduced.

\subsection{Concept Design}

As shown in Figure 1a,b, the main structure of the charging robot consists of 3 serially arranged units, and each unit is a 3UPU parallel mechanism. Thus, this robot belongs to the category of hybrid serial-parallel robots. The first unit is fixed on the robot base, while the charging connector is attached to the last unit. The electrical wire passes through the center of the robot.

For this charging robot, 3UPU mechanism is the key feature. More details can be found in Figure 1c. The 3UPU mechanism is composed of a base platform, a mobile platform, and three identical UPU chains. Each UPU chain consists of two passive universal joints and one active prismatic joint where actuation is applied. It should be noted that the 3UPU mechanism could have different kinds of DOFs according to its structural parameters [32,33], such as 3 rotational (3R) DOFs [34,35], 3 translational (3T) DOFs [36-38], 2 rotational and 1 translational (2R1T) DOFs [39,40]. This paper chooses the 3UPU with 2R1T DOFs, since we think that this configuration is more suitable for the charging application than the other configurations, considering both work space and dexterity. Thus, the proposed charging robot is driven by 9 prismatic joints, and has a total of 9 DOFs including 6 rotations and 3 translations, and controls the 6-DOF end-effector where the charging connector is attached. Therefore, the proposed robot is redundantly actuated and the actuating redundancy is 3 . Through redundant design, the robot can have better dexterity when placing the charging connector in the right pose, especially in confined environment with obstacles. 
Moreover, as shown in Figure 1b, the robot base can move along the rail in the ground. When the current EV is fully charged or meets the preset stopping condition, the robot will stop charging, pull out the connector, and move to the next EV in the charging list. In this way, a single charging robot can manager several EVs. This system can be used in charging stations to decrease long-term occupations and greatly improve efficiency.

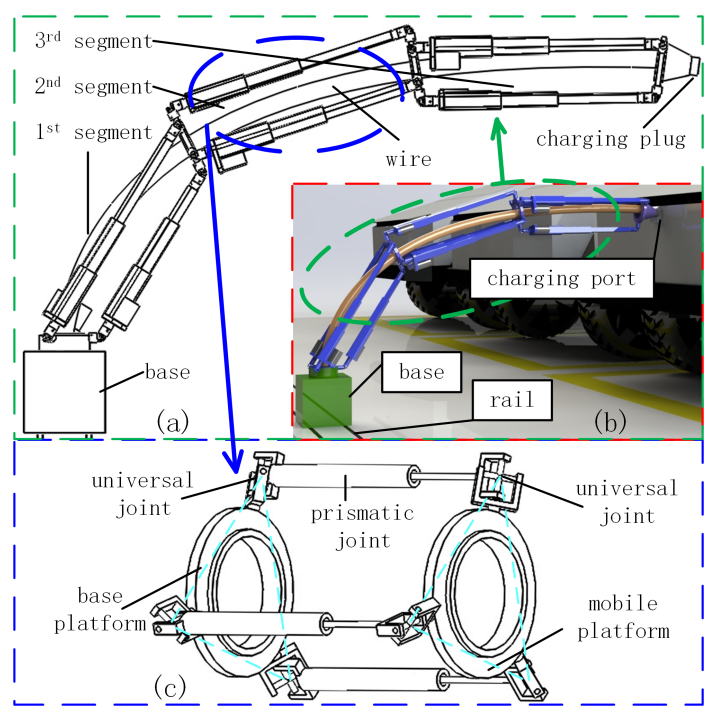

Figure 1. Concept design of the EV charging robot. (a) Structure of the whole robot; (b) assumed charging scenario; (c) schematics of the 3 universal-prismatic-universal (3UPU) mechanism.

\subsection{Kinematic Modeling}

In this section, we address the kinematic model of the charging robot. Without losing generality, the unit number of the robots presented in this section is not limited to 3 , but is assumed to be $n$ where $n$ is an arbitrary positive integer.

As shown in Figure 2, the $i$ th $(i=1,2 \ldots n)$ 3UPU consists of a base platform $O_{i, 0}$, a mobile platform $O_{i, 1}$, and three UPU chains $A_{i, 1} B_{i, 1}, A_{i, 2} B_{i, 2}, A_{i, 3} B_{i, 3}$.

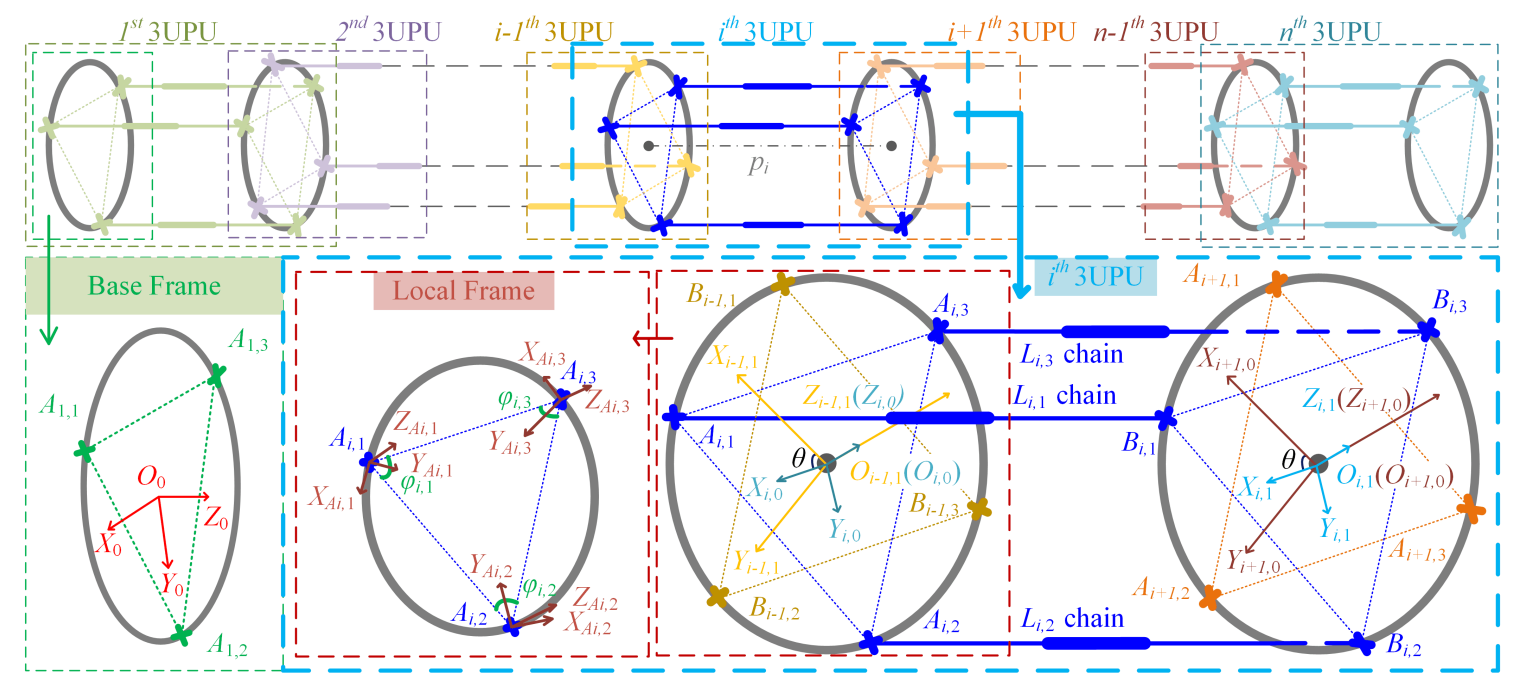

Figure 2. Schematics for the kinematic modeling of the EV charging robot consisting of $n$ units of 3UPU mechanisms.

The $k$ th $(k=1,2,3)$ chain attached the edge of the base platform on $A_{i, k}$ and the edge of the mobile platform on $B_{i, k}$. Each UPU chain consists of one prismatic joint and two universal joints. 
Each universal joint includes two orthogonal revolute joints. For the universal joint at $A_{i, k}$, axis of the 1st revolute joint is perpendicular to $A_{i, k} B_{i, k}$, axis of the the 2nd revolute joint is perpendicular to $A_{i, k} O_{i, 0}$ and the angle between the axis and the base platform is $\delta$. For the universal joint at $B_{i, k}$, axis of the 3 rd revolute joint is perpendicular to $A_{i, k} B_{i, k}$, that is, parallel to the axis of the 1st revolute joint, and axis of the 4 th revolute joint is perpendicular to $B_{i, k} O_{i, 1}$ and the angle between the axis and the mobile platform is $\delta$, the axises of the 2nd and the 4 th revolute joint intersect at point $M_{i, k}$ as shown in Figure 3. $A_{i, 1} A_{i, 2} A_{i, 3}$ and $B_{i, 1} B_{i, 2} B_{i, 3}$ are two congruent regular triangles with $O_{i, 0}$ and $O_{i, 1}$ as their center, respectively. The three chains of the $i$ th 3 UPU unit are parallel with each other at arbitrary configuration.

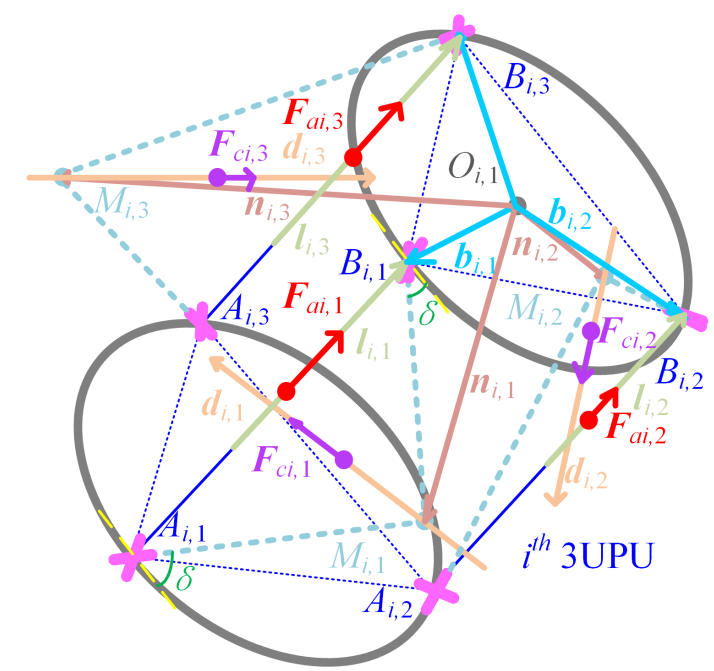

Figure 3. Schematics for the force analysis of the ith 3UPU unit.

To illustrate the kinematics of the robot, following coordinate systems are defined:

1. $\mathcal{R}_{0}\left\{O_{0}, X_{0}, Y_{0}, Z_{0}\right\}$ represents the global frame of the robot. Generally, the origin of the global frame is at the center of the upper surface of the base; $z$-axis is defined perpendicular to the plane of the upper surface of the base; the x-y-plane coincides with the upper surface.

2. $\mathcal{R}_{i, 0}\left\{O_{i, 0}, X_{i, 0}, Y_{i, 0}, Z_{i, 0}\right\}$ represents the local frame of the base platform $A_{i, 1} A_{i, 2} A_{i, 3}$. The origin of the frame is at the center of the platform $O_{i, 0} ; \mathrm{x}$-axis is parallel with the vector pointing from $A_{i, 3}$ to $A_{i, 1} ; \mathrm{z}$-axis is perpendicular to the platform; y-axis is determined by the right-hand rule. It should be noted that the local frame of the base platform of the 1st 3UPU coincides with the global frame of the robot.

3. $\mathcal{R}_{i, 1}\left\{O_{i, 1}, X_{i, 1}, Y_{i, 1}, Z_{i, 1}\right\}$ represents the local frame of the mobile platform $B_{i, 1} B_{i, 2} B_{i, 3}$. The origin point and axis definitions are similar to frame $\mathcal{R}_{i, 0}$.

4. $\mathcal{R}_{A_{i, k}}\left\{O_{A_{i, k}}, X_{A_{i, k}}, Y_{A_{i, k}} Z_{A_{i, k}}\right\}$ represents the local frame of the $k$ th chain of the $i$ th unit. The origin of the frame coincides with point $A_{i, k} ; \mathrm{z}$-axis is parallel with z-axis of frame $\mathcal{R}_{i, 0}$; y-axis coincides with the bisector of angle $A_{i, k} ; \mathrm{x}$-axis is determined by the right-hand rule.

The coordinates of $A_{i, k}(k=1,2,3)$ can be expressed in frame $\mathcal{R}_{i, 0}$ as:

$$
\begin{aligned}
& \mathcal{R}_{i, 0} A_{i, 1}=\left[p \cos \left(\varphi_{i, 2}-\frac{\pi}{2}\right),-p \sin \left(\varphi_{i, 2}-\frac{\pi}{2}\right), 0\right]^{\mathrm{T}} \\
& \mathcal{R}_{i, 0} A_{i, 2}=\left[p \cos \left(3 \varphi_{i, 2}-\frac{\pi}{2}\right), p \sin \left(3 \varphi_{i, 2}-\frac{\pi}{2}\right), 0\right]^{\mathrm{T}} \\
& \mathcal{R}_{i, 0} A_{i, 3}=\left[-p \cos \left(\varphi_{i, 2}-\frac{\pi}{2}\right),-p \sin \left(\varphi_{i, 2}-\frac{\pi}{2}\right), 0\right]^{\mathrm{T}}
\end{aligned}
$$


where

$$
p=\frac{a_{i, 2}}{2 \cos \left(\varphi_{i, 1}+\varphi_{i, 2}-\frac{\pi}{2}\right)}
$$

where $a_{i, 1}$ is the length of $A_{i, 2} A_{i, 3}, a_{i, 2}$ is the length of $A_{i, 1} A_{i, 3}, a_{i, 3}$ is the length of $A_{i, 1} A_{i, 2}$, $\varphi_{i, k},(i=1,2, \ldots, n),(k=1,2,3)$ is the angle of $A_{i, k}$.

Due to the fact that the three chains of the $i$ th 3 UPU unit are parallel with each other all along, the coordinates of points $B_{i, k}$ can be expressed in frame $\mathcal{R}_{A_{i, k}}$ according to the geometrical relationship as $\mathcal{R}_{A_{i, k}} B_{i, k}=\left[x_{i, k}, y_{i, k}, z_{i, k}\right]^{\mathrm{T}}$ where:

$$
\begin{aligned}
& x_{i, k}=\frac{L_{i, k}}{2 \sin \frac{\varphi_{i, k}}{2}}\left(\frac{L_{i, k}-L_{i, k+1}}{2 a_{i, k+2}}-\frac{L_{i, k}-L_{i, k+2}}{2 a_{i, k+1}}\right) \\
& y_{i, k}=\frac{L_{i, k}}{2 \cos \frac{\varphi_{i, k}}{2}}\left(\frac{L_{i, k}-L_{i, k+1}}{2 a_{i, k+2}}+\frac{L_{i, k}-L_{i, k+2}}{2 a_{i, k+1}}\right) \\
& z_{i, k}=\sqrt{L_{i, k}^{2}-x_{i, k}^{2}-y_{i, k}^{2}}
\end{aligned}
$$

where $k=1,2,3$, when $k=2, L_{i, k+2}=L_{i, 1}, a_{i, k+2}=a_{i, 1}$, when $k=3, L_{i, k+1}=L_{i, 1}, a_{i, k+2}=a_{i, 2} . L_{i, k}$ is the length of the $k$ th chain of the $i$ th 3 UPU unit.

For the $i$ th $3 \mathrm{UPU}$ unit, it is easy to obtain the rotation matrix:

$$
\begin{aligned}
& \mathcal{R}_{i, 0} R=R_{Z_{i, 1}}\left(\eta_{i, 1}\right) \\
& \mathcal{R}_{A_{i, 1}} \\
& \mathcal{R}_{i, 0} R=R_{Z_{i, 2}}\left(2 \eta_{i, 1}+\eta_{i, 2}\right) \\
& \mathcal{R}_{A_{i, 2}} \\
& \mathcal{R}_{i, 0} R=R_{Z_{i, 3}}\left(-\eta_{i, 3}\right) \\
& \mathcal{R}_{A_{i, 3}}
\end{aligned}
$$

where $\eta_{i, 1}=\frac{\pi-\varphi_{i, 1}}{2}, \eta_{i, 2}=\frac{\pi-\varphi_{i, 2}}{2}, \eta_{i, 3}=\frac{\pi-\varphi_{i, 3}}{2}$.

Thus, the coordinates of points $B_{i, k}$ can be expressed in frame $\mathcal{R}_{i, 0}$ as:

$$
\mathcal{R}_{i, 0} B_{i, k}={ }_{\mathcal{R}_{i, 0}}^{\mathcal{R}_{i, k}} R \cdot{ }^{\mathcal{R}_{A_{i, k}}} B_{i, k}+{ }^{\mathcal{R}_{i, 0}} A_{i, k}
$$

Then the center point of the mobile platform, i.e., the origin of $\mathcal{R}_{i, 1}$ can be expressed in $\mathcal{R}_{i, 0}$ as:

$$
\mathcal{R}_{i, 0} O_{i, 1}=\frac{\sum_{k=1}^{3} \mathcal{R}_{i, 0} B_{i, k}}{3}
$$

It is easy to get the rotation matrix between $\mathcal{R}_{i, 1}$ and $\mathcal{R}_{i, 0}$, and the distance between $O_{i, 0}$ and $O_{i, 1}$, as:

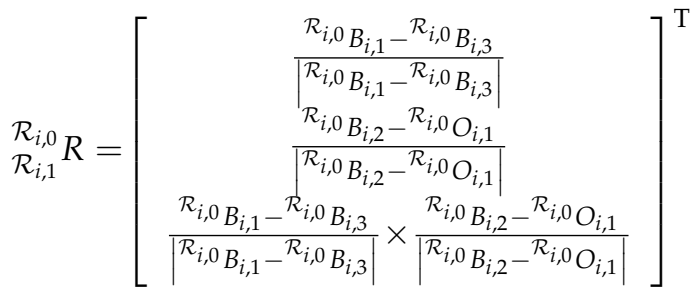

$$
\begin{aligned}
& p_{i}=\left|\mathcal{R}_{i, 0} O_{i, 1}-{ }^{\mathcal{R}_{i, 0}} O_{i, 0}\right|
\end{aligned}
$$

Due to the fact that the frame $\mathcal{R}_{1,0}$ coincides with the frame $\mathcal{R}_{0}$, the homogeneous transformation matrix between $\mathcal{R}_{1,0}$ and $\mathcal{R}_{0}$ can be expressed as:

$$
{ }_{\mathcal{R}_{10}}^{\mathcal{R}_{0}} \mathbf{T}=\mathbf{E}_{4 \times 4}
$$


We assume that the local frame $\mathcal{R}_{i-1,1}$ of the mobile platform of the $(i-1)$ th 3 UPU rotates about $Z_{i-1,1}$ by $\theta$ to get the local frame $\mathcal{R}_{i, 0}$ of the base platform of the $i$ th 3UPU, the rotation matrix between $\mathcal{R}_{i-1,1}$ and $\mathcal{R}_{i, 0}$ is $R_{Z_{i-1,1}}(\theta)$. When $i$ is even, the rotation is in clockwise, $\theta>0$. When $i$ is odd, the rotation is in anticlockwise, $\theta<0$.

The homogeneous transformation matrix between $\mathcal{R}_{i, 0}$ and $\mathcal{R}_{i-1,0}$ can be expressed as:

$$
{ }_{\mathcal{R}_{i-1,0}}^{\mathcal{R}_{i, 0}} \mathbf{T}=\mathcal{R}_{\mathcal{R}_{i-1,0}} \mathbf{T} \cdot{ }_{\mathcal{R}_{i, 0}}^{\mathcal{R}_{i, 1}} \mathbf{T}
$$

where ${ }_{\mathcal{R}_{10}}^{\mathcal{R}_{i-1,1}} \mathbf{T}=\left[\begin{array}{cc}R_{Z_{i-1,1}}(\theta) & 0_{3 \times 1} \\ 0_{1 \times 3} & 0\end{array}\right]$.

Then the homogeneous transformation matrix between $\mathcal{R}_{i, 0}$ and $\mathcal{R}_{0}$ can be expressed as:

$$
{\stackrel{\mathcal{R}}{\mathcal{R}_{i, 1}}}_{\mathcal{R}_{i=1}} \mathbf{T}=\left(\prod_{j=1}^{i} \mathcal{R}_{j-1,0}^{\mathcal{R}_{j, 0}} \mathbf{T}\right) \cdot{ }_{\mathcal{R}_{i, 1}}^{\mathcal{R}_{i, 0}} \mathbf{T}(i=1,2 \ldots n)
$$

where ${ }_{\mathcal{R}_{i, 0}}^{\mathcal{R}_{i-1,0}} \mathbf{T}={ }_{\mathcal{R}_{1,0}}^{\mathcal{R}_{0}} \mathbf{T}$, when $i=1$.

In this case, the position and orientation of the end-effector can be seperately expressed as:

$$
\begin{aligned}
& \mathbf{P}={ }^{\mathcal{R}_{0}} O_{n, 1}={ }_{\mathcal{R}_{n, 1}}^{\mathcal{R}_{0}} \mathbf{T} \cdot{ }^{\mathcal{R}_{n, 1}} O_{n, 1}
\end{aligned}
$$

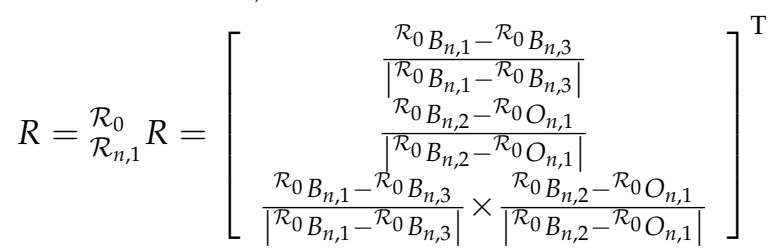

The kinematic model structure in Equations (1)-(19) also matches the modeling results in [39,40].

\section{Static Model and Validation of the EV Charging Robot}

In order to analysis the load capacity of the EV charging robot, dynamic model is necessary. However, the robot motion is usually slow for most charging operations. In this case, static model can be used instead of dynamic model if the inertia forces and the Coriolis forces are neglected. Therefore, this section will present the static modeling and validation of the proposed robot.

\subsection{Static Model}

Based on the principle of virtual work, for the ith 3UPU unit, the formula for solving the statics can be written as:

$$
\boldsymbol{\sigma}_{i}^{\mathrm{T}} \cdot \Delta \boldsymbol{\Theta}_{i}+\mathbf{F}_{i}^{\mathrm{T}} \cdot \Delta \mathbf{X}_{i}=0
$$

where $\varnothing_{i}$ denotes the forces of the joints, $\Delta \boldsymbol{\Theta}_{i}$ denotes the displacements of the joints, $\mathbf{F}_{i}$ denotes the force of the mobile platform, $\Delta \mathbf{X}_{i}$ denotes the displacement of the mobile platform, since:

$$
\Delta \boldsymbol{\Theta}_{i}=\mathbf{J}_{\mathbf{S} i} \cdot \Delta \mathbf{X}_{i}
$$

then:

$$
\boldsymbol{\sigma}_{i}=-\left(\mathbf{J}_{\mathbf{S} i}^{-1}\right)^{\mathrm{T}} \cdot \mathbf{F}_{i}
$$


where the $6 \times 6$ matrix $\mathbf{J}_{\mathbf{S} i}$ is the Jacobian matrix of the $i$ th 3 UPU unit, which can be calculated by:

$$
\mathbf{J}_{\mathbf{S} i}=\left[\begin{array}{cc}
\mathbf{1}_{i, 1}{ }^{\mathrm{T}} & \left(\mathbf{b}_{i, 1} \times \mathbf{1}_{i, 1}\right)^{\mathrm{T}} \\
\mathbf{1}_{i, 2} \mathrm{~T} & \left(\mathbf{b}_{i, 2} \times \mathbf{1}_{i, 2}\right)^{\mathrm{T}} \\
\mathbf{1}_{i, 3} \mathrm{~T} & \left(\mathbf{b}_{i, 3} \times \mathbf{1}_{i, 3}\right)^{\mathrm{T}} \\
\mathbf{d}_{i, 1} \mathrm{~T} & \left(\mathbf{n}_{i, 1} \times \mathbf{d}_{i, 1}\right)^{\mathrm{T}} \\
\mathbf{d}_{i, 2} \mathrm{~T} & \left(\mathbf{n}_{i, 2} \times \mathbf{d}_{i, 2}\right)^{\mathrm{T}} \\
\mathbf{d}_{i, 3} \mathrm{~T} & \left(\mathbf{n}_{i, 3} \times \mathbf{d}_{i, 3}\right)^{\mathrm{T}}
\end{array}\right]
$$

where the $3 \times 1$ vector $\mathbf{1}_{i, k}$ represents the unit vector pointing from $A_{i, k}$ to $B_{i, k}, \mathbf{1}_{i, k}$ can be calculated as:

$$
\mathbf{1}_{i, k}=\frac{\mathcal{R}_{i, 0} B_{i, k}-\mathcal{R}_{i, 0} A_{i, k}}{\left|\mathcal{R}_{i, 0} B_{i, k}-\mathcal{R}_{i, 0} A_{i, k}\right|},(k=1,2,3)
$$

the $3 \times 1$ vector $\mathbf{b}_{i, k}$ represents the vector pointing from $O_{i, 1}$ to $B_{i, k}, \mathbf{b}_{i, k}$ can be calculated as:

$$
\mathbf{b}_{i, k}={ }^{\mathcal{R}_{i, 0}} B_{i, k}-\mathcal{R}_{i, 0} O_{i, 1}
$$

the $3 \times 1$ vector $\mathbf{d}_{i, k}$ represents the unit vector along the axis of the revolute joint attached to the prismatic joint. The $3 \times 1$ vector $\mathbf{n}_{i, k}$ represents the vector from $\mathcal{R}_{i, 0} \mathbf{O}_{i, 1}$ to $\mathcal{R}_{i, 0} \mathbf{M}_{i, k}$, which can be calculated as:

$$
\mathbf{n}_{i, k}=\mathcal{R}_{i, 0} \mathbf{M}_{i, k}-\mathcal{R}_{i, 0} \mathbf{O}_{i, 1},(k=1,2,3)
$$

where $\mathcal{R}_{i, 0} \mathbf{M}_{i, k}$ is the intersection point of the axises of the revolute joints attached to the platforms of the $k$ th chain as shown in Figure 3. There is

$$
\left[\mathcal{R}_{0} \mathbf{v}_{n}, \mathcal{R}_{0} !_{n}\right]^{\mathrm{T}}=\sum_{i=1}^{n} \mathbf{J}_{\mathbf{R} i}\left[\mathcal{R}_{i, 0} \mathbf{v}_{n}, \mathcal{R}_{i, 0} !_{n}\right]^{\mathrm{T}}
$$

where the Jacobian matrix map from the end effector to the mobile platform of the $i$ th 3 UPU can be calculated as: when $i<n$,

$$
\mathbf{J}_{\mathbf{R} i}=\left[\begin{array}{cc}
\mathcal{R}_{1,0} R & \mathbf{J}_{\mathbf{R} i}^{1,2} \\
\mathcal{R}_{i, 0} & \mathcal{R}_{1,0} R \\
0_{3 \times 3} & \mathcal{R}_{i, 0}
\end{array}\right]
$$

where $\mathbf{J}_{\mathbf{R} i}^{1,2}=-\left[\sum_{j=i}^{n-1} \mathbf{S}\left(\begin{array}{l}\mathcal{R}_{1,0} \\ \mathcal{R}_{j+1,0}\end{array} \cdot \mathcal{R}_{j+i, 0} O_{j+1}\right)\right] \cdot \mathcal{R}_{\mathcal{R}_{i, 0}} R$ when $i=n$,

$$
\mathbf{J}_{\mathbf{R} i}=\left[\begin{array}{cc}
\mathcal{R}_{1,0} R & 0_{3 \times 3} \\
\mathcal{R}_{i, 0} & \mathcal{R}_{1,0} R \\
0_{3 \times 3} & \mathcal{R}_{i, 0}
\end{array}\right]
$$

Considering the principle of virtual work, there is:

$$
\mathbf{F}_{\mathbf{S} i}=-\left(\mathbf{J}_{\mathbf{R} i} \cdot \mathbf{J}_{\mathbf{S} i}^{-1}\right)^{\mathrm{T}}\left[\mathcal{R}_{0} \mathbf{F}_{n}, \mathcal{R}_{0} \mathbf{T}_{n}\right]^{\mathrm{T}}
$$

where $\mathbf{F}_{\mathbf{S} i}=\left[F_{a i, 1}, F_{a i, 2}, F_{a i, 3}, F_{c i, 1}, F_{c i, 2}, F_{c i, 3}\right]^{\mathrm{T}}$ represents a six dimensional vector consists of active and constrained forces. $F_{a i, k},(k=1,2,3)$ denotes the active force of the $k$ th prismatic joint of the $i$ th 3UPU unit, its direction is shown in Figure 3. $F_{c i, k}(k=1,2,3)$ denotes the constrained force of the $k$ th prismatic joint of the $i$ th $3 \mathrm{UPU}$ unit, its direction is shown in Figure 3. $\left[\mathcal{R}_{0} \mathbf{F}_{n}, \mathcal{R}_{0} \mathbf{T}_{n}\right]^{\mathrm{T}}$ denotes the force of the end effector expressed in $\mathcal{R}_{0}$.

The static model structure in Equations (20)-(30) also matches the modeling results in [17,41]. 


\subsection{Simulation Validation}

In this section, we first validate the statics model presented in the previous section in Adams. In order to verify the correctness of the statics model, a 3D assembly mechanism with parameters in Table 2 is generated in SolidWorks 2016. It is then imported into Adams and set the motion trajectory of the end-effector of the robot as follows:

$$
\begin{aligned}
& \text { Orientation }:\left\{\begin{array}{l}
\alpha=-\frac{\pi}{2} \\
\beta=0 \\
\gamma=0
\end{array},\right. \\
& \text { Position }:\left\{\begin{array}{l}
X=0 \\
Y=158+3 t, \\
Z=200
\end{array} \quad t=0 \sim 10 s\right.
\end{aligned}
$$

In order to verify the correctness of the statics model presented in the previous section, apply an external force expressed in $\mathcal{R}_{0}$ as $\mathbf{F}_{\mathbf{e x}}=[0,100,0,0,0,0]^{\mathrm{T}}(\mathrm{N})$ to the end-effector of the robot at the center of the mobile platform of the $n$th 3 UPU unit. Coding in Matlab based on the statics model and import the parameters and motion trajectory above into the program, then the active force and constrained force for each chain can be computed. Import the same data into Adams and measure the active force and constrained force of each chain which keep the statics equilibrium. Import the data measured in Adams into Matlab to compare with the computed by the statics model. The results are shown in Figure 4.

It can be seen clearly that the results obtained from Adams measurement and Matlab computation are in good agreement. The maximal value of the absolute force error is $0.001 \mathrm{~N}$, thus the correctness of the statics model is verified. Then the statics model can be used in the following study.
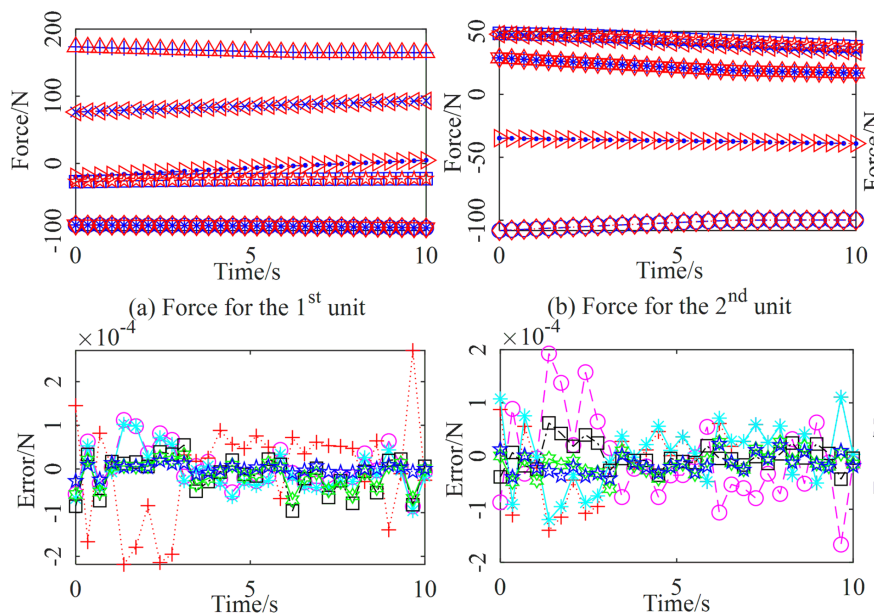

(d) Absolute force error for the $1^{\text {st }}$ unit

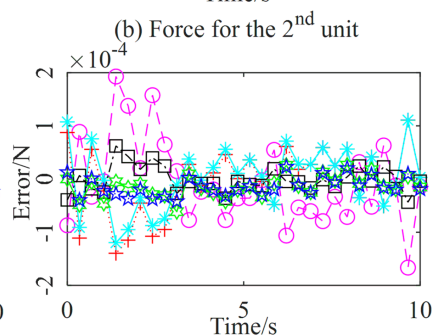

(e) Absolute force error for the $2^{\text {nd }}$ unit

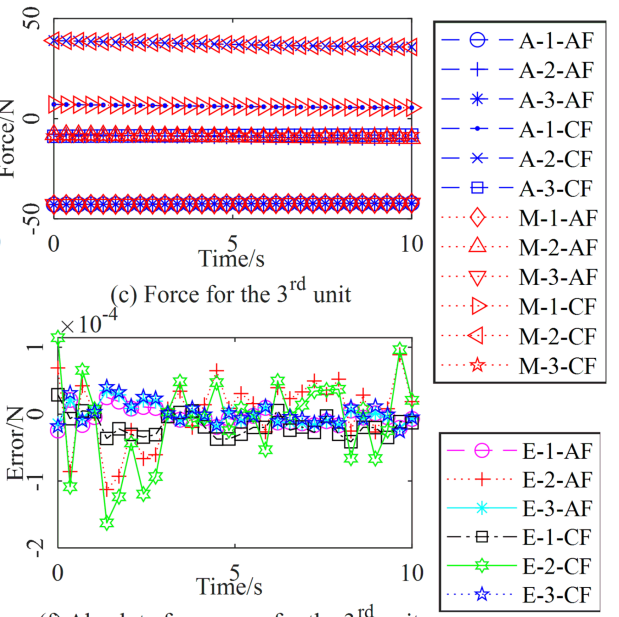

(f) Absolute force error for the $3^{\text {rd }}$ unit

Figure 4. Comparisons between Adams measurement and Matlab calculation ('A' stands for 'Adams measurement', ' $\mathrm{M}$ ' for 'Matlab statics model', ' $\mathrm{E}^{\prime}$ for 'absolute force error' between results obtained from two ways for the same force, 'AF' for 'active force', 'CF' for 'constrained force', the number ' $1,2,3$ ' for the sequence number of the chain. For example, 'A-1-AF' stands for the active force of 1st chain obtained from Adams measurement, and ' $\mathrm{M}-3-\mathrm{CF}^{\prime}$ stands for the constrained force of 3rd chain obtained from Matlab calculation.) 
Table 2. Robot parameters used in the simulation validation ( $i$ denotes the sequence number of the 3UPU unit $1 \leq i \leq 3, k$ denotes the sequence number of the chain $1 \leq k \leq 3$ ).

\begin{tabular}{cccc}
\hline Parameters & Value & Parameters & Value \\
\hline$\theta(\mathrm{rad})$ & $\frac{\pi}{3}$ & $\delta(\mathrm{rad})$ & $\frac{\pi}{4}$ \\
$a_{i, k}(\mathrm{~mm})$ & $60 \sqrt{3}$ & $L_{i, k}(\mathrm{~mm})$ & $70-140$ \\
$\varphi_{i, k}(\mathrm{rad})$ & $\frac{\pi}{3}$ & - & - \\
\hline
\end{tabular}

\section{Load Capacity Analyses and Optimizations}

The load capacity of the EV charging robot is an important characteristic to evaluate the performance of the robot, since the charging connector need enough force to be plugged into the charging port. General, the plugging operation is along the horizontal direction considering the position of the charging port in most EVs. Therefore, in this section we use the horizontal force along a certain trajectory as an index to evaluate the load capacity of the robot.

The trajectory for plugging in and pulling out the charging connector is the same as the one expressed in Section 3.2 (Equations (31) and (32)). The basic structural parameters of the robot are listed in Table 2.

\subsection{Analysis in Term of the Manipulator Configuration}

As a result that the charging robot is redundantly actuated, there exist infinite solutions for a given end-effector pose. Each solution corresponds to a manipulator configuration. This section will analyze the effect of manipulator configuration to the maximum load capacity of the charging robot.

Firstly, two groups of manipulator configurations are calculated using the kinematic model, for the same trajectory of the end-effector given by Equations (31) and (32). Results are shown in Figure 5, where Figure $5 \mathrm{a}-\mathrm{c}$ represent the manipulator's configurations for all the 30 poses along the trajectory, and Figure $5 \mathrm{~d}-\mathrm{f}$ represent the corresponding lengths of the 9 actuating prismatic joints. It is clear to see that the manipulator configurations are different even for the same end-effector trajectory, which can be explained by the actuating redundancy.

After the manipulator configuration is determined, force analysis can be made. For a hybrid serial-parallel robot with $n$ units, there are $6 n+1$ unknowns including $3 n$ actuating forces $F_{a i, k}, 3 n$ constrained forces $F_{c i, k}$ and the horizontal external force $F_{y}$. We can also obtain $6 n$ equations according to the static model expressed by Equation (30). The number of unknowns are more than that of the equations, thus there are infinite solutions that all satisfy the static equilibrium. Optimization can be used to calculate the maximum horizontal external force. Mathematically, this problem can be described as follows.

Objective: Maximize $\left(\left|F_{y}\right|\right)$

Variables: $F_{y}, F_{a i, k}, F_{c i, k}$

Equality Constraints: Static Equations (20)-(30)

Boundaries: $\left|F_{a i, k}\right| \leq 1000 \mathrm{~N},\left|F_{c i, k}\right| \leq 1000 \mathrm{~N}, F_{y}<0 \mathrm{~N}$ 

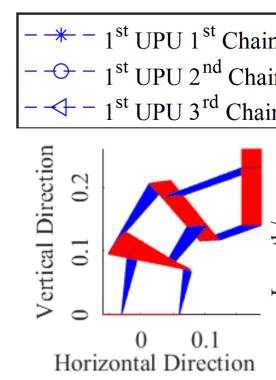

(a)

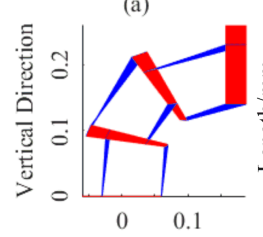

Horizontal Direction

(b)

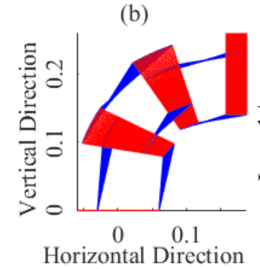

(c)
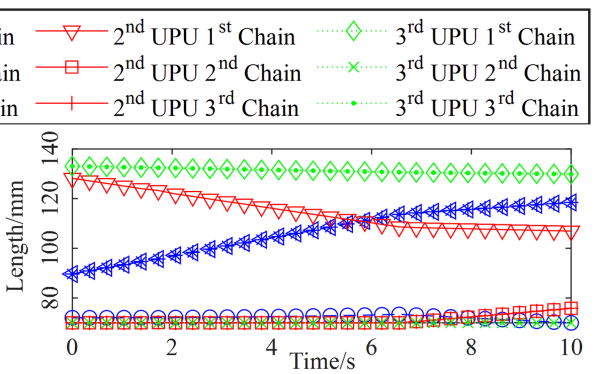

(d)

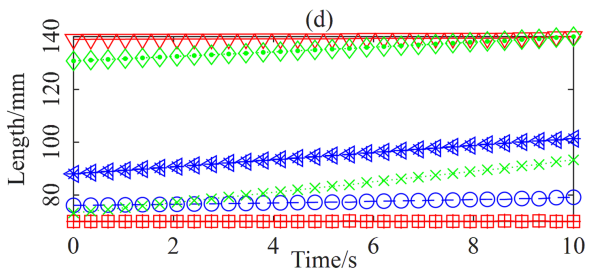

(e)

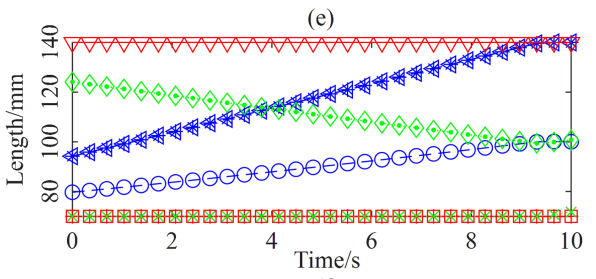

(f)

Figure 5. Configurations and prismatic joint lengths of the robot. (a-c) represent robot configurations along a given end-effector trajectory, and $(\mathbf{d}-\mathbf{f})$ represent the actuating prismatic joint lengths corresponding to configurations $(\mathbf{a}-\mathbf{c})$, where $(\mathbf{a}, \mathbf{d})$ show the results for a random configuration, $(\mathbf{b}, \mathbf{e})$ for another random configuration, $(\mathbf{c}, \mathbf{f})$ for the optimized configuration.

In this paper, the Matlab (Matlab is a software by Mathworks company.) function 'fmincon' is used to solve the above optimization problem. Results for the two groups of manipulator configurations are shown in Figure 6 by red plus sign and black cross. It is shown that the maximum horizontal forces are different for these two groups of manipulator configurations. In fact, these two groups of configurations are just random ones from the infinite kinematic solutions. Therefore, our objective in this section is to find a best group of manipulator configurations that could maximize the horizontal external force. Mathematically, this problem can be described as follows.

Objective: Maximize $\left(\left|F_{y}\right|\right)$

Variables: $F_{y}, F_{a i, k}, F_{c i, k}, L_{i, k}$

Equality Constraints: Kinematic Equations (1)-(19) and static Equations (20)-(30)

Boundaries: $\left|F_{a i, k}\right| \leq 1000 \mathrm{~N},\left|F_{c i, k}\right| \leq 1000 \mathrm{~N}, F_{y}<0 \mathrm{~N}, 70 \leq L_{i, k} \leq 140 \mathrm{~mm}$

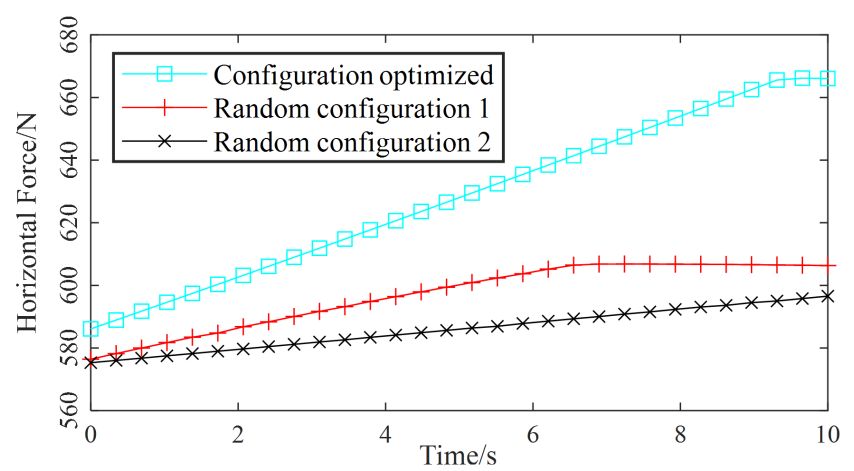

Figure 6. Comparison of the maximum reachable horizontal forces obtained by different configurations along the same given trajectory. 
Compared with previous optimization problem, there are $3 n$ more unknowns $L_{i, k}$, but only 6 equations are added according to the kinematic model. In total, there are $9 n+1$ unknowns and $6 n+6$ equations. For a charging robot with 3 units, the number of unknowns is 28 , while the number of equations is 24 . Similar as precious optimization, fmincon function is also used to solve this problem. Results are shown in Figure 6 by cyan square. As we can see, the maximum horizontal forces are enlarged for all the end-effector poses in the trajectory after configuration optimization. Compared to other random configurations, the maximum increment is $70.55 \mathrm{~N}$, and the average increment is $36.60 \mathrm{~N}$. To sum up, the manipulator configuration has obvious effect on the load capacity of the EV charging robot. The maximum horizontal force applied to the end-effector could be obviously enlarged by optimize the manipulator configuration.

\subsection{Analysis in Term of the Unit Length}

In previous Section 4.1, each unit of the proposed EV charging robot has the same initial length. To answer the question whether unit length would affect the robot load capacity, this section will analyze the effect of unit length on the maximum horizontal force applied to the end-effector.

Firstly, different groups of unit lengths are used to calculate the maximum horizontal force. In order to make the comparison reasonable, the total initial length of the robot keeps constant when varying the initial length of each unit. Specifically, the initial length of the first unit is $70+\Delta L \mathrm{~mm}$, and the second unit is $70 \mathrm{~mm}$, and the third unit is $70-\Delta L \mathrm{~mm}$. In addition, the boundaries of these three units are $70+\Delta L-140+2 \Delta L \mathrm{~mm}, 70-140 \mathrm{~mm}$, and 70- $\Delta L-140-2 \Delta L \mathrm{~mm}$. Five groups of initial unit lengths can be obtained when $\Delta L$ is respectively $-20,-10,0,10$, and $20 \mathrm{~mm}$. Results are shown in Figure 7. It is obvious that the maximum horizontal forces are quite different for robot with 5 groups of initial unit lengths. In addition, the maximum horizontal forces along the whole trajectory will be enlarged as $\Delta L$ increases. This means that it is effective to improve the load capacity by choosing longer prismatic joint for the base unit in the robot parameter design.

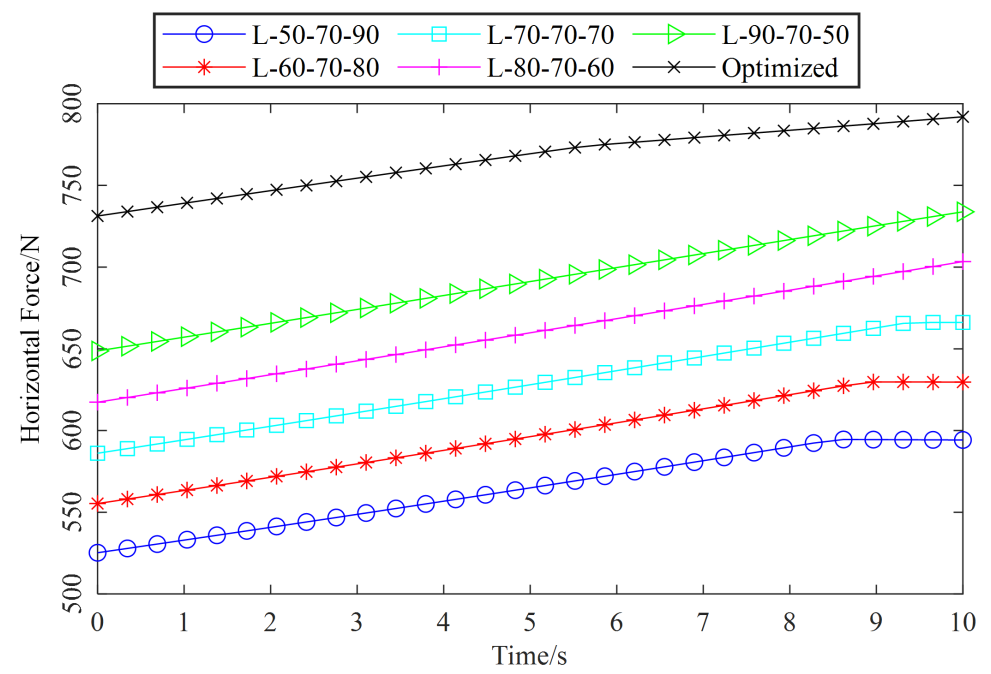

Figure 7. Comparison of the maximum reachable horizontal forces obtained by different distributions of the unit lengths along the same given trajectory. (' $\mathrm{L}$ ' denotes 'length', and the numbers after $\mathrm{L}$ represent the initial lengths of each unit. For example, 'L-50-70-90' represents that the initial length of the 1 st, 2 nd, and 3rd units are respectively 50, 70, and $90 \mathrm{~mm}$.)

Secondly, optimization is made in order to choose the best value of $\Delta L$. The objective of this optimization is to maximize the horizontal force applied to the end-effector. The variables are the horizontal force $F_{y}$, the actuating lengths of each prismatic joint $L_{i, k}$, the variation of the initial prismatic joint lengths $\Delta L$, the actuating forces $F_{a i, k}$, and the constrained forces $F_{c i, k}$. The constraints are the kinematic and static equations. Mathematically, this optimization problem can be described as follows. 
Objective: Maximize $\left(\left|F_{y}\right|\right)$

Variables: $F_{y}, L_{i, k}, \Delta L, F_{a i, k}, F_{c i, k}$

Equality Constraints: Kinematic Equations (1)-(19) and static Equations (20)-(30)

Boundaries: $\left|F_{a i, k}\right| \leq 1000 \mathrm{~N},\left|F_{c i, k}\right| \leq 1000 \mathrm{~N}, F_{y}<0 \mathrm{~N}, 70 \leq L_{i, k} \leq 140 \mathrm{~mm},|\Delta L|<50 \mathrm{~mm}$.

Similar to previous optimizations, the Matlab function fimicon is also used to solve the above problem. Results show that the optimized value of $\Delta L$ is $47.5 \mathrm{~mm}$ when the horizontal forces are maximized. Detail value of the force is shown in Figure 7 by the black line. Compared to the robot with equal unit lengths, the load capacity of the EV charging robot is improved by about $22.02 \%$ after optimizing the unit length.

\subsection{Analysis in Term of the Unit Diameter}

This section will address the effect of unit diameter to the load capacity of the charging robot. Similar to the method used in Section 4.2, 5 groups of diameters are firstly employed in the calculation of the maximum horizontal force applied to the end-effector. Then, optimization is made to determine the best unit diameter that can maximize the robot load capacity.

Supposing that the second unit of the robot has a basic diameter of $120 \mathrm{~mm}$, the first unit's diameter is $120+\Delta D \mathrm{~mm}$, and the third unit is $120-\Delta D \mathrm{~mm}$. With this arrangement, the average diameter of the robot keeps unchanged, thus making the comparison more reasonable. Five different groups of unit diameters are obtained when $\Delta D$ is respectively $-20,-10,0,10,20 \mathrm{~mm}$. For each group of unit diameters, the maximum horizontal forces along the trajectory are calculated. Results are shown in Figure 8. On one hand, the load capacity varies with the unit diameter. On the other hand, there is a obvious trend that the load capacity could be improved by enlarge the diameter of the first unit which closes to the robot base, and vice versa. Therefore, it is efficient to increase the first unit's diameter to improve robot load capacity in the robot design.

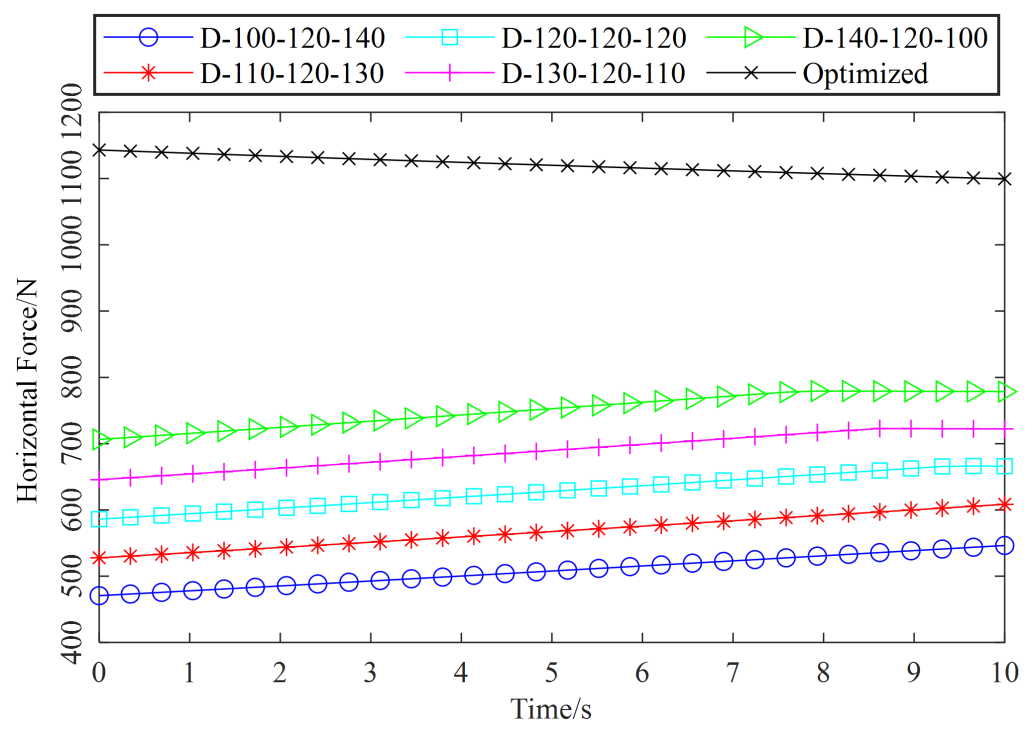

Figure 8. Comparison of the maximum reachable horizontal forces obtained by different distributions of the unit diameters along the same given trajectory. (' $\mathrm{D}$ ' denotes 'diameter', and the numbers after $\mathrm{D}$ represent the diameters of each unit. For example, 'D-100-120-140' represents that the diameters of the 1 st, 2 nd, and 3rd units are respectively 100, 120, and $140 \mathrm{~mm}$.)

Then, optimization is made to find the best value of $\Delta D$ with the objective of maximizing the horizontal force applied to the end-effector. Mathematically, this optimization problem can be described as follows. 
Objective: Maximize $\left(\left|F_{y}\right|\right)$

Variables: $F_{y}, L_{i, k}, \Delta D, F_{a i, k}, F_{c i, k}$

Equality Constraints: Kinematic Equations (1)-(19) and static Equations (20)-(30)

Boundaries: $\left|F_{a i, k}\right| \leq 1000 \mathrm{~N},\left|F_{c i, k}\right| \leq 1000 \mathrm{~N}, F_{y}<0,70 \leq L_{i, k} \leq 140 \mathrm{~mm},|\Delta D|<120 \mathrm{~mm}$.

This optimization problem is also solved by the Matlab function fmincon. Results show that the horizontal force reaches to the peak when $\Delta D$ is $91.42 \mathrm{~mm}$. Further details can be also find in Figure 8 . It is shown that the load capacity increases by about $78.77 \%$ after optimization, compared to the robot with constant unit length.

\subsection{Analysis in Term of the Unit Actuating Force}

This section will analyze the effect of actuating force to the robot load capacity. The analysis procedures are quit similar with the above two sections. The variables are the boundaries of the actuating forces of each unit.

Firstly, a variable $\Delta F$ is defined to vary the actuating force boundaries. This boundary is $-1000-\Delta F \mathrm{~N}-+1000+\Delta F \mathrm{~N}$ for the first unit, and $-1000 \mathrm{~N}-+1000 \mathrm{~N}$ for the second unit, and $-(1000-\Delta F) \mathrm{N}-+(1000-\Delta F) \mathrm{N}$ for the third unit. We can also obtain 5 groups of boundaries by assigning $-200,-100,0,100,200$ to $\Delta F$, respectively. For each group of force boundaries, the maximum horizontal forces applied to the end-effector are calculated along the whole trajectory. Results are shown in Figure 9, where we can see obvious variation in the objective force for different actuating force boundaries. Moreover, the robot load capacity trends to be enlarged as the actuating force boundary of the first unit increases. Further optimization can be made to determine the most suitable value for $\Delta F$. Mathematically, this optimization problem can be described as follows.

Objective: Maximize $\left(\left|F_{y}\right|\right)$

Variables: $F_{y}, L_{i, k}, \Delta F, F_{a i, k}, F_{c i, k}$

Equality Constraints: Kinematic Equations (1)-(19) and static Equations (20)-(30)

Boundaries: $\left|F_{a i, k}\right| \leq 1000 \mathrm{~N},\left|F_{c i, k}\right| \leq 1000 \mathrm{~N}, F_{y}<0,70 \leq L_{i, k} \leq 140 \mathrm{~mm},|\Delta F|<1000 \mathrm{~N}$.

Solving this optimization problem using the Matlab function fmincon, results show that the optimized $\Delta F$ is $500 \mathrm{~N}$. Compared to the robot with constant actuating force boundaries, the objective horizontal forces increase by about $49.95 \%$ after optimization, which can be see in Figure 9. 


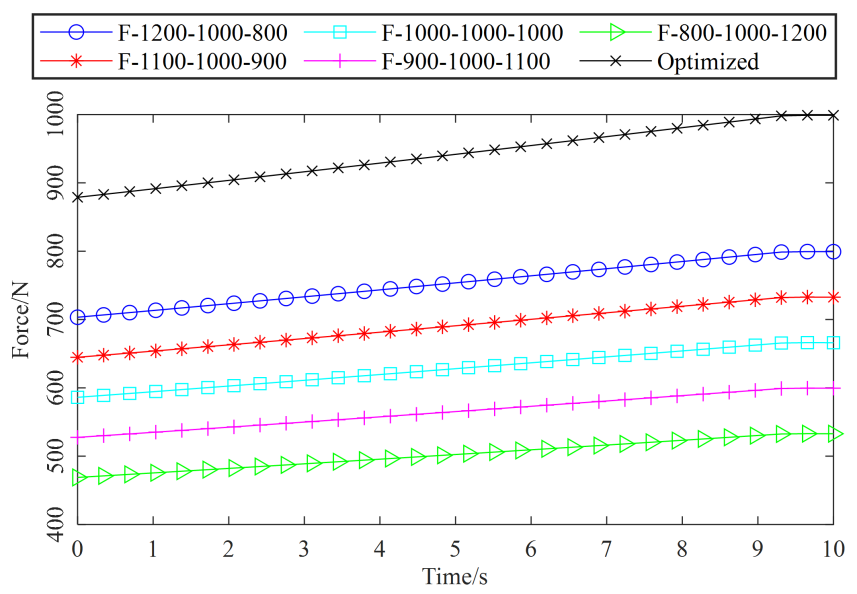

Figure 9. Comparison of the maximum reachable horizontal forces obtained by different distributions of the unit actuating forces along the same given trajectory. (' $\mathrm{F}$ ' denotes 'actuating forces', and the numbers after F represent the force constraints of each unit. For example, 'F-1200-1000-800' represents that the actuating force ranges of the 1 st, 2 nd, and 3 rd units are respectively $-1200 \sim 1200,-1000 \sim 1000$, and $-800 \sim 800$ N.)

\subsection{Multi-Variable Optimization}

According to the above sections, we find that the manipulator configuration and the robot structural parameters such as the unit length, unit diameter and the actuating force boundary, all have obvious effect to the robot load capacity. This section will consider these variables in the same time, and propose a comprehensive optimization algorithm to maximize the objective forces as much as possible.

The multi-variable optimization algorithm can be described as follows.

Objective: Maximize $\left(\left|F_{y}\right|\right)$

Variables: $F_{y}, L_{i, k}, F_{a i, k}, F_{c i, k}, \Delta L, \Delta D, \Delta F$

Equality Constraints: Kinematic Equations (1)-(19) and static Equations (20)-(30)

Boundaries: $\left|F_{a i, k}\right| \leq 1000 \mathrm{~N},\left|F_{c i, k}\right| \leq 1000 \mathrm{~N}, F_{y}<0,70 \leq L_{i, k} \leq 140 \mathrm{~mm},|\Delta L|<50 \mathrm{~mm}$, $|\Delta D|<120 \mathrm{~mm},|\Delta F|<1000 \mathrm{~N}$.

Multi-variable optimization results show that the robot load capacity reaches the peak when $\Delta L$ is $47.5 \mathrm{~mm}, \Delta D$ is $45.43 \mathrm{~mm}$, and $\Delta F$ is $499.47 \mathrm{~N}$. Moreover, as shown in Figure 10, the maximum horizontal forces can be enlarged by about $124.32 \%$ compared to the result obtained by only configuration optimization, and be enlarged by about $130.91 \%$ compared to the result obtained by a random configuration without any optimization. 


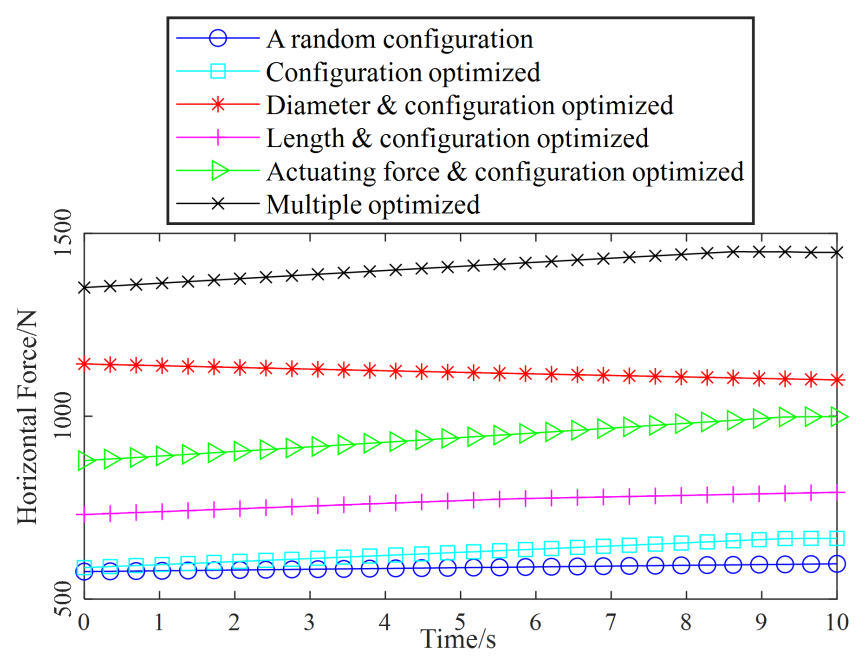

Figure 10. Comparison of the maximum reachable horizontal forces obtained by different optimization solvers along the same given trajectory.

\section{Conclusions}

This paper presented the concept design of a novel hybrid serial-parallel robot for EV charging. The proposed robot mainly contains 3 units of 3 UPU parallel mechanisms that are arranged in serial. We focused on the load capacity analyses of the charging robot, which is important but often neglected in previous researches. For this purpose, the kinematic and static models of the robot were deduced and validated by simulation. Then, we used the maximum reachable horizontal force applied to the end-effector as an index to evaluate and analyze the load capacity of the proposed charging robot.

Results showed that the load capacity is not only associated with the manipulator configuration, but also related to the structural parameters such as the length and diameter of each 3UPU unit, and also limited by the actuating ability of each unit. Moreover, the load capacity exhibits an obvious increasing trend when augmenting the length, and/or the diameter, and/or the actuating force of the robot's first unit, i.e., the unit closed to the base. Further multi-variable optimization manifested that the robot load capacity could be improved by about $131 \%$, compared to the result of a random chosen configuration without any optimization.

The results of this paper could be helpful for the design of hybrid serial-parallel EV charging robot. Future work includes the prototype manufacture and experimental validations.

Author Contributions: Conceptualization, L.Z.; methodology, H.Y. and Q.W.; software, Q.W.; resources, L.Z. and H.Y.; writing — original draft preparation, H.Y. and Q.W.; writing—review and editing, H.Y., Q.W., and L.Z.; supervision, L.Z.; funding acquisition, L.Z. and H.Y.. All authors have read and agreed to the published version of the manuscript.

Funding: This work was supported by the National Natural Science Foundation of China (Grant No. 61803125), the National Natural Science Foundation of Guangdong Province China (Grant No. 2018A030313247), the Basic Research Program of Shenzhen (Grant No. JCYJ20180507183644237, JCYJ20190806144416980, \& JCYJ20190809110415177), and the Youth Innovation Fund of Shenzhen Polytechnic (Grant No. 6019310011K).

Conflicts of Interest: The authors declare no conflict of interest.

\section{Abbreviations}

The following abbreviations are used in this manuscript:

DOF degrees of freedom

UPU a chain consists of a universal joint on each side of a prismatic joint 


\section{Nomenclature}

$n$ the number of 3UPU the robot consists

$i \quad$ the sequence number of 3UPU unit

$k$ the sequence number of UPU chain

$\mathcal{R}_{0} \quad$ the global frame of the robot

$\mathcal{R}_{i, 0} \quad$ the local frame of the base platform of the $i$ th 3UPU

$\mathcal{R}_{i, 1} \quad$ the local frame of the mobile platform of the $i$ th 3 UPU

$\mathcal{R}_{A_{i, 0}} \quad$ the local frame of the $k$ th chain of the $i$ th 3 UPU

$\theta \quad$ the rotation angle between $\mathcal{R}_{i, 0}$ and $\mathcal{R}_{i-1,1}$

${ }_{\mathcal{R}_{p}}^{\mathcal{R}_{q}} \mathbf{T} \quad$ the homogeneous transformation matrix from $\mathcal{R}_{p}$ to $\mathcal{R}_{q}$

$p_{i} \quad$ the distance between $O_{i, 1}$ and $O_{i, 0}$

$\mathcal{R}_{i, 0} O_{i, 1} \quad$ the coordinate of $O_{i, 1}$ expressed in $\mathcal{R}_{i, 0}$

$A_{i, k} \quad$ attached point of the $k$ th chain with the base platform in the $i$ th 3 UPU

$B_{i, k} \quad$ attached point of the $k$ th chain with the mobile platform in the $i$ th 3UPU

$a_{i, k} \quad$ the length of the edge of the triangle on the opposite side of the point $A_{i, k}$

$D_{i} \quad$ the diameter of the $i$ th 3UPU

$\delta$ the angle between the axises of the revolute joints attached to the platform and the plane of the platform

$\varphi_{i, k} \quad$ the angle of $A_{i, k}$

$L_{i, k} \quad$ the length of the $k$ th chain of the $i$ th 3 UPU

$\mathbf{P} \quad$ the position of the end-effector

$\mathbf{R}$ the orientation of the end-effector

$\mathcal{R}_{i, 0} \mathbf{v}_{i} \quad$ the instantaneous velocity of the mobile platform of the $i$ th 3UPU expressed in $\mathcal{R}_{i, 0}$

$\mathcal{R}_{i, 0} !_{i} \quad$ the angular velocity of the mobile platform of the $i$ th 3 UPU expressed in $\mathcal{R}_{i, 0}$

$\mathbf{J}_{\mathbf{S} i} \quad$ the Jacobian matrix of the $i$ th $3 \mathrm{UPU}$

$\mathbf{1}_{i, k} \quad$ the unit vector from $A_{i, k}$ to $B_{i, k}$

$\mathbf{b}_{i, k} \quad$ the vector from $O_{i, k}$ to $B_{i, k}$

$\mathbf{d}_{i, k} \quad$ the unit vector along the axis of the revolute joint attached to the prismatic joint

$\mathcal{R}_{i, 0} M_{i, k}$ the intersection point of the axises of the revolute joints attached to the platforms on the $k$ th chain

$\boldsymbol{\sigma}_{i} \quad$ the force of the joint

$\Delta \boldsymbol{\Theta}_{i} \quad$ the displacement of the joint

$\mathbf{F}_{i} \quad$ the force of the mobile platform

$\Delta \mathbf{X}_{i} \quad$ the displacement of the mobile platform

$\mathbf{J}_{\mathbf{i} i} \quad$ the Jacobian matrix from the end-effector to the mobile platform of the $i$ th 3 UPU

$F_{a i, k} \quad$ the active force of the $k$ th prismatic of the $i$ th $3 \mathrm{UPU}$

$F_{c i, k} \quad$ the constrained force of the $k$ th prismatic of the $i$ th $3 U P U$

${ }^{\mathcal{R}_{0}} \mathbf{F}_{n} \quad$ the force of the end effector expressed in $\mathcal{R}_{0}$

$\mathcal{R}_{0} \mathbf{T}_{n} \quad$ the torque of the end effector expressed in $\mathcal{R}_{0}$

\section{References}

1. Taylor-Haw, C.K.; Haw, B.; Simmons, G.C. Electric Vehicle Charging Station. US Patent 8,487,584, 16 July 2013.

2. Liu, Z.; Wen, F.; Ledwich, G. Optimal planning of electric-vehicle charging stations in distribution systems. IEEE Trans. Power Deliv. 2012, 28, 102-110. [CrossRef]

3. Yamashita, T.; Jodo, K. Charger for an Automobile. US Patent 29/411,436, 15 January 2013.

4. Wu, D.; Aliprantis, D.C.; Gkritza, K. Electric energy and power consumption by light-duty plug-in electric vehicles. IEEE Trans. Power Syst. 2010, 26, 738-746. [CrossRef]

5. Walzel, B.; Sturm, C.; Fabian, J.; Hirz, M. Automated robot-based charging system for electric vehicles. In 16. Internationales Stuttgarter Symposium; Springer: Wiesbaden, Germany, 2016; pp. 937-949.

6. Long, Y.; Wei, C.; Cao, C.; Hu, X.; Zhu, B.; Long, F. Design of high-power fully automatic charging device. In Proceedings of the 2019 IEEE Sustainable Power and Energy Conference (iSPEC), Beijing, China, 21-23 November 2019; pp. 2738-2742.

7. Kong, P.-Y. Autonomous robot-like mobile chargers for electric vehicles at public parking facilities. IEEE Trans. Smart Grid 2019, 10, 5952-5963. [CrossRef] 
8. Lv, X.; Chen, G.; Hu, H.; Lou, Y. A robotic charging scheme for electric vehicles based on monocular vision and force perception. In Proceedings of the 2019 IEEE International Conference on Robotics and Biomimetics (ROBIO), Dali, China, 6-8 December 2019; pp. 2958-2963.

9. Briot, S.; Bonev, I.A. Are parallel robots more accurate than serial robots? Trans. Can. Soc. Mech. Eng. 2007, 31, 445-455. [CrossRef]

10. Liu, T.; Mu, Z.; Wang, H.; Xu, W.; Li, Y. A cable-driven redundant spatial manipulator with improved stiffness and load capacity. In Proceedings of the 2018 IEEE/RSJ International Conference on Intelligent Robots and Systems (IROS), Madrid, Spain, 1-5 October 2018; pp. 6628-6633.

11. Tang, L.; Wang, J.; Zheng, Y.; Gu, G.; Zhu, L.; Zhu, X. Design of a cable-driven hyper-redundant robot with experimental validation. Int. J. Adv. Robot. Syst. 2017, 14, 1729881417734458. [CrossRef]

12. Mu, Z.; Yuan, H.; Xu, W.; Hu, Z.; Liu, T.; Liang, B. Simultaneous Planning Method Considering Both Overall Configuration and End Pose for Hyper-Redundant Manipulators. IEEE Access 2019, 7, 136842-136854. [CrossRef]

13. $\mathrm{Xu}, \mathrm{W} . ; \mathrm{Liu}, \mathrm{T}$;; Li, Y. Kinematics, dynamics, and control of a cable-driven hyper-redundant manipulator. IEEE/ASME Trans. Mechatron. 2018, 23, 1693-1704. [CrossRef]

14. Yuan, H.; Li, Z. Workspace analysis of cable-driven continuum manipulators based on static model. Robot. Comput.-Integr. Manuf. 2018, 49, 240-252. [CrossRef]

15. Yuan, H.; Zhou, L.; Xu, W. A comprehensive static model of cable-driven multi-section continuum robots considering friction effect. Mech. Mach. Theory 2019, 135, 130-149. [CrossRef]

16. Yuan, H.; Chiu, P.W.Y.; Li, Z. Shape-Reconstruction-Based Force Sensing Method for Continuum Surgical Robots With Large Deformation. IEEE Robot. Autom. Lett. 2017, 2, 1972-1979. [CrossRef]

17. Hu, B.; Yu, J.; Lu, Y.; Sui, C.; Han, J. Statics and stiffness model of serial-parallel manipulator formed by $\mathrm{k}$ parallel manipulators connected in series. J. Mech. Robot. 2012, 4, 021012. [CrossRef]

18. He, J.; Zheng, H.; Gao, F.; Zhang, H. Dynamics and control of a 7-dof hybrid manipulator for capturing a non-cooperative target in space. Mech. Mach. Theory 2019, 140, 83-103. [CrossRef]

19. Ibrahim, O.; Khalil, W. Inverse and direct dynamic models of hybrid robots. Mech. Mach. Theory 2010, 45, 627-640. [CrossRef]

20. Rahmani, A.; Ghanbari, A.; Pedrammehr, S. Kinematic analysis for hybrid 2-(6-upu) manipulator using wavelet neural network. In Advanced Materials Research; Trans Tech Publ: Stafa-Zurich, Switzerland, 2014; Volumn 1016, pp. 726-730.

21. Staicu, S. Dynamics modelling of a stewart-based hybrid parallel robot. Adv. Robot. 2015, 29, 929-938. [CrossRef]

22. Merlet, J.-P. Parallel Robots; Springer Science \& Business Media: Berlin, Germany, 2006.

23. Yuan, H.; Courteille, E.; Deblaise, D. Static and dynamic stiffness analyses of cable-driven parallel robots with non-negligible cable mass and elasticity. Mech. Mach. Theory 2015, 85, 64-81. [CrossRef]

24. Yuan, H.; Courteille, E.; Gouttefarde, M.; Hervé, P.E. Vibration analysis of cable-driven parallel robots based on the dynamic stiffness matrix method. J. Sound Vib. 2017, 394, 527-544. [CrossRef]

25. Zi, B.; Wang, N.; Qian, S.; Bao, K. Design, stiffness analysis and experimental study of a cable-driven parallel 3d printer. Mech. Mach. Theory 2019, 132, 207-222. [CrossRef]

26. Romdhane, L. Design and analysis of a hybrid serial-parallel manipulator. Mech. Mach. Theory 1999, 34, 1037-1055. [CrossRef]

27. Castelli, G.; Ottaviano, E.; Ceccarelli, M. A fairly general algorithm to evaluate workspace characteristics of serial and parallel manipulators. Mech. Based Des. Struct. Mach. 2008, 36, 14-33. [CrossRef]

28. Zhao, C.; Guo, H.; Liu, R.; Deng, Z.; Li, B.; Tian, J. Actuation distribution and workspace analysis of a novel 3 (3rrls) metamorphic serial-parallel manipulator for grasping space non-cooperative targets. Mech. Mach. Theory 2019, 139, 424-442. [CrossRef]

29. Guo, H.; Zhao, C.; Li, B.; Liu, R.; Deng, Z.; Tian, J. A transformation method to generate the workspace of an n (3rrs) serial-parallel manipulator. J. Mech. Des. 2019, 141, 092301. [CrossRef]

30. Nayak, A.; Caro, S.; Wenger, P. Kinematic analysis of the 3-rps-3-spr series-parallel manipulator. Robotica 2019, 37, 1240-1266. [CrossRef]

31. Arora, R.; Agarwal, D.; Bera, T.K. Workspace analysis and trajectory tracking of a planar hybrid manipulator with ball screw feed drive. J. Braz. Soc. Mech. Sci. Eng. 2019, 41, 378. [CrossRef] 
32. Huang, Z.; Li, Q. General methodology for type synthesis of symmetrical lower-mobility parallel manipulators and several novel manipulators. Int. J. Robot. Res. 2002, 21, 131-145. [CrossRef]

33. Huang, Z.; Li, S.; Zuo, R. Feasible instantaneous motions and kinematic characteristics of a special 3-dof 3-upu parallel manipulator. Mech. Mach. Theory 2004, 39, 957-970. [CrossRef]

34. Zheng, X.; Luo, Y.; Deng, Z.; Bin, H. Statics of rotational 3-upu parallel mechanisms based on principle of virtual work. In Proceedings of the 2007 IEEE International Conference on Robotics and Biomimetics (ROBIO), Sanya, China, 15-18 December 2007; pp. 1954-1959.

35. Di Gregorio, R. Kinematics of the 3-upu wrist. Mech. Mach. Theory 2003, 38, 253-263. [CrossRef]

36. Di Gregorio, R.; Parenti-Castelli, V. Mobility analysis of the 3-upu parallel mechanism assembled for a pure translational motion. J. Mech. Des. 2002, 124, 259-264. [CrossRef]

37. Tsai, L.-W.; Joshi, S. Kinematics and optimization of a spatial 3-upu parallel manipulator. J. Mech. Des. 2000, 122, 439-446. [CrossRef]

38. Zheng, X.; Deng, Z.; Luo, Y.; Bin, H. Static analysis of translational 3-upu parallel mechanism based on principle of virtual work. in Global Design to Gain a Competitive Edge; Springer: London, UK, 2008; pp. 681-690.

39. Chen, Z.; Zhang, Y.; Huang, K.; Huang, Z. Symmetrical 2r1t parallel mechanism without parasitic motion. J. Mech. Eng. 2016, 52, 9-15. [CrossRef]

40. Chen, Z.; Liu, X.; Zhang, Y.; Huang, K.; Huang, Z. Dynamics analysis of a symmetrical 2r1t 3-upu parallel mechanism. J. Mech. Eng. 2017, 53, 46-53. [CrossRef]

41. Joshi, S.A.; Tsai, L.-W. Jacobian analysis of limited-dof parallel manipulators. J. Mech. Des. 2002, 124, $254-258$. [CrossRef]

(C) 2020 by the authors. Licensee MDPI, Basel, Switzerland. This article is an open access article distributed under the terms and conditions of the Creative Commons Attribution (CC BY) license (http:/ / creativecommons.org/licenses/by/4.0/). 